\title{
Prismas trasescalares en el estudio de las migraciones ${ }^{1}$
}

\author{
Trans-scalar prisms in the study of migrations
}

\author{
F. Javier GARCÍA CASTAÑO \\ Aurora ÁLVAREZ VEINGUER \\ María RUBIO GÓMEZ \\ Instituto de Migraciones, Universidad de Granada \\ fjgarcia@ugr.es \\ auroraav@ugr.es \\ mariarubio@ugr.es
}

Recibido: 6 de mayo de 2011

Aceptado: 30 de mayo de 2011

\section{Resumen}

El presente artículo pretende reflexionar sobre lo que la antropología social puede aportar al estudio de las migraciones y, a la vez, "descubrir" lo que puede aprender también la disciplina de este campo de estudio, y, de esta manera transdisciplinar, sus saberes. De aquí aprenderemos lo importante que es para el estudio de las migraciones el aquí y allí no como situaciones o lugares, sino como nociones que construyen y reconstruyen los procesos de identificación. Desde ellos trataremos de mostrar varios ejemplos de nuestras investigaciones etnográficas, que nos pongan en la situación de analizar el concepto de contexto desde sus dimensiones relacionales. Es una noción de contexto que no debe dimensionarse en lo espacial, sino en la dotación de significados de los acontecimientos sociales y culturales que deben ser inscritos en él. De todo ello surgirá el enfoque transescalar, que en un contexto global debe tomar la antropología en el estudio de cualquier fenómeno sociocultural, lo que le obligará a intensificar una etnografía multisituada.

Palabras clave: Migraciones, etnografia multisituada, enfoque transescalar.

\begin{abstract}
This article aims to reflect on the way social anthropology can contribute to the study of migrations and, simultaneously, to "find out" what the discipline can also learn from this field of study, and thus to transfer its knowledge to other areas. From here we will learn how important here and there are for the study of migrations, not as situations or places, but as

${ }^{1}$ El presente texto es un conjunto de reflexiones que son deudoras de varias experiencias investigadoras de diferentes investigadores e investigadoras del Laboratorio de Estudios Interculturales. En concreto, tienen que ver con el proyecto "Diffusion and context of transnational migrant organizations in Europe" (TRAMO), del que es investigadora principal Aurora Álvarez Veinguer, y con los proyectos: "Integración de escolares denominados 'inmigrantes': relaciones entre el éxito-fracaso escolar y las relaciones familia-escuela" (SEJ2007-67155/SOCI) y "Multiculturalidad e integración de población inmigrante extranjera en las escuelas andaluzas" (P06-HUM-02380), de los que es investigador principal F. Javier García Castaño.
\end{abstract}


constructed and reconstructed notions of identification processes. From that point, we will try to show some examples of our ethnographic research that lead to the analysis of the concept of situation from its relational dimension. A notion of context that shouldn't be seen in relation to space, but in the provision of meanings for the social and cultural events within it. Therefore a trans-scalar approach of anthropology will arise in a global context and referring to any sociocultural pnenomenon. Such an approach wil lead to the enforcement of a multisited ethnography.

Keywords: Migrations, Multi-sited Ethnography, Trans-scalar Approach.

Referencia normalizada: García Castaño, F. J. \& Álvarez Veinguer, A. \& Rubio Gómez, M. (2011). Prismas trasescalares en el estudio de las migraciones. Revista de Antropología Social, 20, 203-228.

SUMARIO: 1. Introducción. 2. ¿Qué puede aportar la antropología social al estudio de los procesos migratorios? 3. Punto de partida: devenir (in)cierto de la antropología social. Una oportunidad para la transdisciplinariedad. 4. Algunas traducciones desde diferentes experiencias investigadoras. Primeras tentativas hacia el prisma transescalar. 5. Breve coda. 6. Referencias bibliográficas.

\section{Introducción}

Cuando un estudiante de antropología social lee la introducción de la obra de Malinowski Los argonautas del Pacifico Occidental, además de tomar una relativa distancia con la dimensión de "aventura" en la que el autor sitúa su acción —en especial, si es capaz de comprender que está leyendo una obra escrita en 1922 con datos producidos en la década anterior durante su experiencia de campo, ya mítica para la antropología social, en la isla Mailu, en el Sur de la actual Papua Nueva Guinea-, empieza a comprender un cierto aislamiento y práctica individualista en los estudios que está realizando. Por si al empezar la lectura queda duda sobre el particular, basta con llegar al también mítico párrafo en el que se comienza a explicar qué es el trabajo de campo²: "imagínese que de repente está en tierra, rodeado de todos sus pertrechos, solo en una playa tropical...". Individualismo y aventura en el quehacer etnográfico están servidos.

Parece fácil observar, tras la lectura de esa monografía y de sus sucesoras en forma y principios, que la antropología y sus métodos remiten tradicionalmente al estudio de micro sociedades que son delimitadas por y para la investigación antropológica. El resultado aparente es que las personas que nos dedicamos a la antropología, en el sentido que surge de la experiencia malinowskiana, terminamos estu-

${ }^{2}$ Debe recordarse, como se hace en la edición de este capítulo introductorio de Malinowski en el recopilatorio de Honorio Velasco (1993), que la mayoría de los encabezamientos que se leen ahora en dicha introducción no aparecen en la obra original. En este caso, cuando Malinowski describía los primeros pasos entre los "indígenas", no aparecía titulado originalmente como "trabajo de campo", según ahora sí podemos leerlo. 
diando una "comunidad", haciendo trabajo de campo en una comunidad y sobre una comunidad. Pero las consecuencias tienen un mayor alcance epistemológico, pues muchos de esos antropólogos y antropólogas acabamos indicando en nuestros respectivos curriculum, que estudiamos tal o cual lugar y no que estudiamos en tal o cual lugar; es decir, convertimos el contexto-lugar en objeto de estudio, en el peor de los casos, o hacemos del contexto un lugar irrenunciable, vinculado a nuestro objeto de estudio.

Precisamente esta cuestión es la que pretendemos problematizar en las siguientes páginas. Haremos ver como el contexto debe dejar de ser, si es que alguna vez lo fue, un lugar, para convertirse sobre todo en (re)presentaciones de situaciones relacionales - transescalares-, en las cuales se inscriben los fenómenos socioculturales que pretendemos interpretar, y no en términos geográficos, donde actúan y se relacionan los actores sociales con los que estudiamos los asuntos que investigamos.

Podemos y debemos simplificar la reflexión de la que partimos para hacernos entender. Las metas del trabajo etnográfico, tal como nos las recordaba Malinowski en la introducción ya citada - "organización" y "anatomía" de la comunidad, él decía de la cultura, "vida real" y "comportamientos" más "mentalidad indígena"-, nos conducen con mucha facilidad a una investigación de un grupo humano que, por la propia dinámica del estudio, terminamos aislando del resto del mundo. Verdad es que desde entonces ha llovido mucho y no es tan común este tipo de planteamientos en los quehaceres etnográficos actuales, pero sus influencias se mantienen si recordamos la importancia que se le da a los lugares estudiados para muchos antropólogos y antropólogas.

El valor que se le da a la "elección del campo" parece fácil de justificar por la necesidad que tiene todo proceso interpretativo en la antropología social de ser inscrito en un lugar, de colocar la acción en un "marco de intenciones" y de saber las "condiciones" de la misma: "Cuando un agente dice o hace algo, lo importante no es sólo destacar el contenido de esa práctica, sino también dar cuenta del marco de intenciones y de condiciones en que tal práctica ha sido llevada a cabo (Velasco y Díaz de Rada, 1997: 220).

Pero ello no debe hacer que el lugar se convierta en el LUGAR. Ello está muy próximo, si no es idéntico, a lo que Beck ha planteado el "nacionalismo metodológico". Muchas etnografías han construido con el lugar del trabajo de campo el contexto y con ello lo han esencializado hasta hacer de él un territorio epistemológico, donde sólo debía haber un recurso metodológico. Lo estudiado no son los lugares, lo que se hace es estudiar en los lugares o con los lugares - si queremos referirnos finalmente a las gentes que habitan un determinado sitio-. Dicho en otras palabras, la antropología no puede confinar los saberes que produce a los lugares donde se realizan los estudios, dado que las fronteras que pretendíamos inscribir para tales lugares no existen ni en términos sociales (Beck, 2000) ni en términos culturales

${ }^{3}$ Por limitaciones de espacio en el presente artículo, no vamos a problematizar y analizar el concepto de comunidad, que ha sido ya discutido en profundidad en el contexto de la Antropología Social. Pero no podemos dejar de subrayar su caracter ciertamente polifacético. 
(Díaz de Rada, 2010). Pensar que etnografiar un determinado lugar es terminar por inscribir en dicho territorio un cultura, es parte del espejismo del que pretendemos hablar y se relaciona muy directamente con esa visión de la modernidad en la que la sociedad queda inscrita en los confines del Estado-nación.

De la misma manera que Wimmer y Glick Schiller $(2002,2003)$ nos indican que no se puede seguir asumiendo que las fronteras territoriales de un Estado-nación son las que delimitan las unidades de análisis en los estudios que realizamos en las ciencias sociales, nosotros pretendemos mostrar como el estudio de las migraciones se ha convertido en una temática privilegiada, para mostrar que el trabajo de campo no puede continuar situado en un lugar, y debe comenzar a pensarse en múltiples lugares interralacionados, en los que inscribir los significados de los contextos que estudiamos.

... los enfoques de las migraciones que habían predominado hasta la década de los noventa centrados únicamente en lo que ocurría en la sociedad receptora de la migración adolecían de este llamado nacionalismo metodológico eludiendo así considerar la complejidad del fenómeno migratorio y las prácticas y procesos que se desarrollan más allá de los límites del Estado-nación. Como han señalado algunos autores, este nacionalismo metodológico en el estudio de las migraciones internacionales ha sido producido no sólo por la ceguera teórica y metodológica de los investigadores, sino también por el intento de conciliar sus intereses con los de las agencias estatales o financiadoras de los países receptores... (Moraes, 2010: 35).

En cierto modo, pretendemos mostrar como su superación es también un avance para los estudios antropológicos, si se está dispuesto a esta multilocalización del trabajo de campo, construyendo el contexto como una noción y no simplemente como un lugar.

Esta idea de multilocalización o multisituación no es nueva en la antropología social. Marcus (1995) lo explica con la movilidad de la persona investigadora entre diferentes lugares, actores y posiciones sociales. Pero no se trata de una nueva versión del método comparativo, según lo usaban evolucionistas o difusionistas, ni de una fase de investigación como es planteada en los estudios transculturales ${ }^{4}$. Localizar el trabajo de campo es más que comparar lo que ocurre en diferentes lugares, es trascender la tradición disciplinar y pensar el contexto más allá de los lugares concretos, observando que sólo cobran significado los acontecimientos socioculturales cuando los tradicionales lugares del trabajo de campo se ensanchan y toman como territorio la globalidad en la que dichos actores sociales interactúan. Pero no nos confundamos, tampoco nos referimos con nuestra posición a un nuevo matiz sobre una visión completa de lo que pueda ocurrir en "nuestro contexto" mirando más allá para entender el más acá. No se trata de un matiz de la posición holística en la

${ }^{4}$ Hannerz (2003) critica la novedad de esta etnografía multisituada al hacer ver que no existe invención en esa idea de comparar varias localizaciones en el trabajo de campo. Él insiste en que la novedad debe ser vista en la capacidad que tengamos en etnografíar los vínculos y relaciones que se establecen entre esos lugares distantes espacialmente, pero unívocamente unidos para entender, explicar e interpretar los objetos teóricos de estudio. 
investigación antropológica, que plantea el clásico argumento de saber enciclopédico, como se nos proponía en la visión de cultura de Tylor. No se trata de llegar a todo, sino de inscribir en los múltiples lugares los significados de los acontecimientos sociales. Se trata de (re)construir el contexto desde sus múltiples significados. Se trata de experimentar con nuevas formas, de aproximarnos a los significados no cediendo ante posibles experimentaciones que puedan romper la ortodoxia disciplinar. Se trata, incluso, de indisciplinar los procedimientos en la producción del saber con nuevas experimentaciones, alejándonos de simples etnografías realistas que desdibujan las complejidades de los fenómenos estudiados con la aparente pretensión de su descripción:

Además, la occidentalización es una noción demasiado simple de cambio cultural. Hoy se requieren nuevo motivos narrativos, de manera que la etnografía ha de ser experimental, es decir, ha de abrir nuevos caminos, probar nuevas formas de discurso correspondientes a las circunstancias cambiantes de los pueblos y de las culturas, de los grupos sociales y de las comunidades, y al continuo redescubrimiento que unos hacen de otros, reinventando imágenes de sí mismos y de los otros a medida que se ven afectados por nuevos cambios (Velasco y Díaz de Rada, 1997: 82).

Como nos recuerdan los ya citados Velasco y Díaz de Rada (1997: 221): "para un etnógrafo, la localización de la información ha de implicar la mayor cantidad de elementos situacionales posibles", pero de ello no debe transcenderse que tal localización se encuentra ubicada en un solo lugar físico. Al final, de lo que se trata es de, a través de los individuos, "decir cosas sobre las situaciones" y hablar de las situaciones es "hablar de las relaciones" que se generan y (re)producen en diferentes contextos.

Pero insistimos, no es novedad absoluta lo que aquí planteamos, sino incidencia sobre lo que nuevos saberes pueden aportar a las críticas y crisis de la antropología. Por sólo citar algunos ejemplos dentro de las prácticas etnográficas, próximos a las reflexiones antropológicas y moviéndonos, como aquí queremos, en el estudio de las migraciones, merecen alguna mención breve ciertos estudios recientes. Por ejemplo, Semin (2009) reflexiona sobre este concepto de "etnografía multilocalizada" cuestionando la noción de territorio para este tipo de investigaciones, en especial en el estudio de las sociedades postcoloniales. En especial, se preocupa de las redes sociales que se tejen entre gentes que viven separadas espacialmente, pero que ello no les impide constituir una comunidad - trasnacional, menciona ella - utilizando determinadas formas ceremoniales en los intercambios. Por su parte, Autant-Dorier (2009) termina haciendo una defensa de lo que denomina identidad en movimiento en su estudio de poblaciones turcas a lo largo de varios años y en diferentes lugares: las críticas aquí son desde las propias herramientas que utilizamos para las observaciones de los fenómenos que pretendemos interpretar, los recursos teóricos recurrentes, hasta las categorías de análisis — parentesco, cultura, tradición, integración, etc.-.

Un ejemplo más cercano, realizado en el propio Laboratorio de Estudios Interculturales de la Universidad de Granada, al que pertenecemos, lo conforma el 
estudio sobre la posibilidad de constituir una comunidad transnacional en el caso de los migrantes uruguayos en España. En sus manifestaciones metodológicas, la autora nos deja clara esta posición multilocalizada o multisituada:

Esta metodología permite acercarse a los fenómenos sociales que se construyen a través de relaciones entre diversos lugares y localidades y que no podrían ser abordados con una etnografía tradicional. Este trabajo no implica un mero estudio comparativo de diferentes lugares sino que se centra en las relaciones que se establecen entre los mismos (Moraes, 2010: 3).

Las preguntas que Natalia Moraes se formula a la hora de enfrentarse con sus intereses de estudio fijan con claridad su posición, que reconociendo el valor del estudio en un contexto particular le obligan a replantear, como nosotros queremos aquí, la noción de contexto:

Ahora bien, ¿cuál es el contexto de un estudio qué busca conocer las prácticas
transnacionales? ¿Qué desafíos implica para la etnografía una investigación que
pretende dentro del eje espacial incorporar diferentes niveles como el local, el na-
cional y el transnacional? Estas preguntas planteadas al inicio de la investigación
me llevaron a modificar completamente mi estrategia metodológica, que siguió ba-
sándose en la etnografía, pero pasó a convertirse en lo que se ha llamado una etno-
grafía multisituada o multilocalizada (Moraes, 2010: 292; citando a Marcus, 1995).

Nuestra pretensión es ampliar este marco de debate, y reflexionando sobre lo que la antropología puede aportar al estudio de las migraciones, "descubrir" lo que puede aprender también la disciplina de este campo de estudio y, de esta manera transdisciplinar, sus saberes. De aquí aprenderemos lo importante que es para el estudio de las migraciones el aquí y allí no como situaciones o lugares, sino como nociones que construyen y reconstruyen los procesos de identificación y desde ellos trataremos de mostrar varios ejemplos de nuestras investigaciones etnográficas, que nos pongan en la situación de analizar el concepto de contexto desde sus dimensiones relacionales. Es una noción de contexto que no debe dimensionarse en lo espacial, sino en la dotación de significados de los acontecimientos sociales y culturales que deben ser inscritos en él.

\section{2. ¿Qué puede aportar la antropología social al estudio de los procesos migratorios?}

En el contexto del Estado español y desde los pasados años ochenta se han multiplicado de forma exponencial investigaciones, tesis doctorales, seminarios, programas de estudios de postgrado, congresos y publicaciones sobre procesos migratorios con un singular foco de atención desde la antropología social, aunque el fenómeno de las migraciones, y en especial el de la inmigración en España, se ha configurado como un claro ámbito de trabajo interdisciplinar y en algunos casos empieza incluso a ser superado como conocimiento propio de una disciplina.

Políticas migratorias, relaciones de alteridad generadas en el país de llegada y en el de origen, relaciones interculturales, educación y migración, género y migración, 
redes migratorias, prácticas transnacionales, procesos de reunificación familiar, participación, trabajo doméstico, políticas de integración, mediación intercultural o gestión de la diversidad: son algunas de las múltiples temáticas que están siendo abordadas en las últimas décadas desde diferentes enfoques bajo el paraguas de la antropología social. Es evidente que la producción no representa un corpus homogéneo, sin embargo, a pesar de una aparente sobresaturación temática en torno al estudio de las migraciones, la desatención epistemológica (Santamaría, 2008) y, consecuentemente, metodológica sobre el estudio de los procesos migratorios sigue siendo una realidad vigente. Coincidimos con Gregorio (2009), en que sería necesario no dejar de replantearnos y cuestionarnos el modo en cómo continuamos construyendo la mayoría de las narraciones, amparados en muchos casos, bajo el perturbador y maniqueo prisma del "problema social"s.

Quizás podría intuirse que el estudio de las migraciones por la antropología social responde a una secuencia lógica, condicionada por la crisis disciplinaria y la propia reformulación del trabajo de campo en las últimas décadas. Los clásicos "otros", que vivían en lugares remotos y lejanos, han tendido a borrarse o desaparecer del "interés académico", al ser sustituidos por los denominados "otros", que nos son más cercanos e incluso habitan entre "nosotros". Sin embargo, ambos escenarios: monopolio de la otredad, y crisis disciplinaria y sustitución de los "otros", parecerían argumentos insuficientes para indagar sobre las aportaciones que subyacen al quehacer antropológico en el estudio de las migraciones ${ }^{6}$.

Qué duda cabe de que la antropología social establece una relación casi directa entre el estudio de las migraciones y lo que han sido, consideramos, sus temáticas centrales desde hace tiempo: alteridad, diferencia y diversidad cultural. En el momento en que el migrante es pensado y narrado como un "otro", la antropología social ha encontrado suficiente abono para cultivar y desarrollar su producción científica. Sin embargo, no podemos dejar de subrayar que, en términos generales, la antropología social no se ha dedicado realmente al estudio de los sujetos migrantes y las migraciones hasta hace relativamente poco ${ }^{7}$. Más aún, ha prestado

\footnotetext{
${ }^{5}$ Para analizar la construcción de la migración como problema social, existe numerosa literatura, pero sobre todo se recomienda mirar Santamaría (2002), Agrela (2006) y Gil (2006).

${ }^{6}$ Es verdad que otras "otredades" para el caso de España han estado presentes en las posibilidades de los estudios antropológicos, pero no han llegado a tener el mismo predicamento en la antropología en España el estudio de los mundos rurales que el estudio de las comunidades gitanas; éstas últimas han representado con claridad "nuestra otredad étnica".

${ }^{7}$ Merecen una especial atención, en este sentido, las aportaciones que realiza Steven Vertovec (2007) en la introducción al monográfico dedicado a la antropología de las migraciones de la revista Ethnic and Racial Studies. El autor plantea que vivimos en un momento en que el auge del estudio de las migraciones desde la antropología social - que siempre ha sido un tema central y a la vez marginal en el desarrollo de la antropología social (Eades, 1987 en Vertovec, 2007) — es indiscutible. Vertovec rescata las aportaciones de la escuela de Manchester, que desde 1930 trabajaba sobre las dinámicas migratorias y su impacto en las sociedades receptoras, aunque este hecho supone una excepción, dado que, como reconoce el mismo autor, la antropología social ha prestado mucho más atención a los patrones de orden social que se daban en el seno de las sociedades y grupos humanos que a los procesos de desintegración y cambio — en los que las migraciones son fundamentales - . Al revisar la literatura internacional, coincidimos con Vertovec (2007) en plantear el auge de los estudios sobre etnicidad y
} 
reducida atención a las acciones colectivas de los migrantes, entendidas éstas en términos de sujetos políticos (Peró, 2009) ${ }^{8}$. Como subraya Peró, el hecho de ser sujetos poco reconocidos en muchas ocasiones, con escasos derechos políticos, ha promovido desde los paradigmas más estructuralistas no profundizar en los procesos de agenciamiento de los migrantes, no reconociéndolos como sujetos políticos. Como contrapeso, debemos reconocer ahora que la juventud de estos estudios aún no ha permitido que la antropología social figure como argumentario en las agendas internacionales que se preocupan del fenómeno de las migraciones (Martín Castro, 2007).

Desde estas páginas, aunque siendo conscientes de las limitaciones y deficiencias que existen, nos resulta sugerente - y nos parece necesario- indagar sobre las potencialidades que la propia situacionalidad - margen en el centro- de la antropología social puede aportar al estudio de las migraciones, especialmente a través de sus propuestas conceptuales y metodológicas. Esta situacionalidad, condicionada y vertebrada por un casi permanente estado de crisis, la coloca en un lugar posiblemente privilegiado para abordar el estudio de las migraciones. Si dirigimos una mirada exhaustiva al estudio de las migraciones, podemos destacar que en términos generales, aunque las aportaciones desde la antropología social son cada vez mayores, no es un campo disciplinario hegemónico en el conjunto de las investigaciones y en la producción científica, singularmente dentro del Estado español ${ }^{9}$. Sus aportaciones escasas y su frecuente interés descriptivo por las circunstancias concretas y

construcción de la identidad de las poblaciones migrantes en los países de destino en los pasados años 70; temática abordada por Cohen (1969), Graves y Graves (1974), Peterson (1972) o Mayer (1971), entre otros. Sus estudios se alimentan de la famosa aportación de Frederick Barth, Ethnic Groups and Boundaries (1969). A partir de 1990, surge la perspectiva transnacional — según una exhaustiva revisión de literatura, llevada a cabo por Gustavo Cano (2005), entre finales de la década de los ochenta y el año 2003 se publicaron más de 1.300 artículos en ciencias sociales, centrados en el transnacionalismo o la perspectiva transnacional - y el giro en los estudios de las migraciones se hace evidente en el seno de la antropología social, que va introduciendo nuevas formas de mirar y entender los procesos migratorios y a sus protagonistas.

${ }^{8}$ En términos generales, Peró (2009) critica que las acciones colectivas de los migrantes han sido poco analizadas y estudiadas desde los estudios multidisciplinares de las migraciones.

${ }^{9}$ En dicho contexto contamos con un importante grupo de investigadores/as, repartidos por diferentes universidades españolas y departamentos de antropología que, desde sus grupos de investigación y/o en colaboración con otros, mantienen la producción científica sobre las migraciones desde la antropología social. En la Universidad Autónoma de Barcelona, destacamos los trabajos del Grupo EMIGRA, liderado por Silvia Carrasco, así como los de su compañero de departamento y miembro del Grupo de Investigación sobre Migraciones (GRM) Dan Rodríguez. En la Universidad Rovira i Virgili de Tarragona, encontramos a Juan Josep Pujadas; en la Universidad de Girona y en colaboración con otras disciplinas - pedagogía o psicología —, destacamos el trabajo de Carles Serra. No podemos dejar de mencionar los trabajos de Adela Franzé de la Universidad Complutense de Madrid, así como los de Carlos Giménez, Director del Instituto Universitario de Investigación sobre Migraciones, Etnicidad y Desarrollo Social (IMEDES) y del Programa "Migración y Multiculturalidad" de la Universidad Autónoma de Madrid. En esta misma universidad, destacamos los trabajos de Liliana Suárez, Coordinadora del Grupo de Investigación Estudios en Migración, Identidad, y Ciudadanía (EMIC), y de Ángeles Ramírez. Mirando hacia el sur, encontramos a Francisco Checa y Ángeles Arjona, miembros del grupo de investigación Laboratorio de Antropología Social y Cultural de la Universidad de Almería; en la 
puntuales - micro - han dificultado generalmente su participación en la producción metateórica; ¡que, a fin de cuentas, es lo que cuenta! Es casi seguro que el interés relativo que la etnografía — uno de los principales andamiajes metodológicos de la antropología social — ha ejercido en el panorama de las ciencias sociales en general, y en el estudio de las migraciones en particular, ha condicionado estar, en gran medida, en lo que podríamos considerar los "márgenes del centro". Sin embargo, intuimos que su permanente ejercicio de cuestionar, indagar y tratar de poner a conversar a diferentes universos de sentidos y significados ${ }^{10}$-recurriendo en muchos casos a la experimentación metodológica y a la búsqueda de equilibrios teóricos entre las explicaciones estructurales y los prismas focalizados en los distintos actores y sus capacidades de agencia-, la convierte en un campo expectante para los tiempos venideros. Eso resultará posible siempre y cuando seamos capaces de abandonar ciertas herencias anacrónicas ${ }^{11}$, que no pueden adaptarse al ritmo vertiginoso de las transformaciones del presente. En gran medida, entendemos que sólo los espacios capaces de redefinirse, y con voluntad de re-inventarse y cuestionarse ${ }^{12}$, disponen de la potencia para despojarse de lo aprendido y desarrollar herramientas y marcos conceptuales lo suficientemente flexibles que permitan trascender lo evidente para conocer otros modos de construir sentido.

Desde nuestra forma de entender la antropología social, la preocupación no debe ser una u otra forma de producir conocimiento, sino el significado de los conocimientos producidos. Sin duda, la forma en la producción de los conocimientos es importante y relevante para la preocupación de los significados, pero no puede ni debe alcanzar a éstos en importancia, si no queremos convertir a la antropología social en una disciplina permanentemente preocupada por su sentir y ser metodológico. Nuestro compromiso debe ser con la replicación de los fenómenos que estudiamos, cuestión diferente es que marquemos con claridad el rigor de los caminos seguidos.

Sin embargo, no queremos confundir, porque no reivindicamos desde estas páginas una seña identitaria propia y consecuentemente excluyente, a través de arcaicas y obsoletas estrategias que ponen en comparación conocimientos parcelados enfatizando la mayor o menor idoneidad de cada uno de ellos para el estudio de diferentes fenómenos sociales. Tampoco se persigue producir un discurso romanticista y caduco que aspira a seguir justificando exclusividades más que complementariedades. Sin embargo, sí nos parece interesante y necesario indagar sobre qué puede aportar la antropología social al estudio de los procesos migratorios desde prismas transescalares y transdisciplinares.

Universidad de Sevilla, a Emma Martín; y en la Universidad de Granada al equipo del Laboratorio de Estudios Interculturales y del recién creado Instituto de Migraciones.

${ }^{10}$ Más allá de la diferenciación etic y emic.

${ }^{11}$ Entendemos por herencias anacrónicas: seguir en el siglo XXI apelando al canon ortodoxo y propio de la antropología social de los años cuarenta del siglo XX.

${ }^{12}$ Generalmente es condición favorable para el que habita la periferia del centro. No podemos negar que la antropología social dentro de las ciencias sociales nunca ha optado por un lugar hegemónico ni por una butaca en el palco de honor. 


\section{Punto de partida: devenir (in)cierto de la antropología social. Una opor- tunidad para la transdisciplinariedad}

Ginrich (2010) comienza su artículo "Transitions: Notes on Sociocultural Anthropology's Present and its Transnational Potential" haciendo una interesante reflexión sobre la temática en torno a la que han girado dos de los eventos antropológicos internacionales relevantes en los últimos años. Por una parte, menciona la 108 reunión anual de la Asociación Antropológica Americana, realizada en Filadelfia en 2009, y, por otro lado, la celebración en 2008 del "Jensen Memorial Lecture Series", llevada a cabo en Frankfurt. Ambos eventos giraron en torno al posible final de la antropología social. Más allá del predecible tono pesimista y/o incluso apocalíptico, Ginrich subraya como ejes centrales del debate de ambos eventos internacionales el cuestionarse, por una parte, el lugar que ocupa en la actualidad la antropología $\mathrm{y}$, consecuentemente, por otra parte, plantearse el futuro de la antropología social. Los términos eran: ¿qué es lo siguiente que viene ahora? Ginrich en este artículo analiza la situación actual de la antropología social ${ }^{13} \mathrm{y}$ sus concretas potencialidades de transnacionalización y del tránsito de encontrarse precisamente entre un estadio de desaparición y emergencia.

A lo largo de las páginas, se analizan las diferentes transiciones que han sucedido en la antropología social como consecuencia del pensamiento crítico y de la auto-reflexión —neo-marxismo, feminismo, posmodernidad, poscolonialidad - de la propia disciplina en las últimas décadas. En gran medida, para el autor la actual transición se caracteriza por el abandono de la era nacional, caracterizada por las tradiciones nacionales - en términos epistemológicos, conceptuales, temáticos y metodológicos-, produciéndose un giro hacia las investigaciones transnacionales y globales $^{14}$. De un modo importante, el nuevo tránsito histórico supone una respuesta hacia la hegemonía anglo-americana y la disolución de las metanarrativas nacionales (Restrepo y Escobar, 2005; citado en Gingrich, 2010: 555).

Dos son principalmente, según Gingrich, las potencialidades que sugieren seguir avanzando en el nuevo transito histórico: a) la larga tradición en la antropología social de explicar acontecimientos macro a partir de análisis micro, y b) las pasadas décadas de crítica y auto-reflexión que han venido trabajando sobre los caminos que habría que adoptar en el futuro.

A modo de síntesis, el autor reivindica apostar por la dimensión transnacional y el abandono de la hegemonía Euro-Americana. Si bien la primera propuesta parece ser cada vez más compartida y aceptada dentro de las investigaciones antropológicas ${ }^{15}$, la segunda tan sólo parece ocupar el centro de atención de los posicionamientos más

${ }^{13}$ En términos del autor sería "antropología sociocultural".

${ }^{14}$ Ello es lo que Gingrich denomina como segunda transición histórica. La primera transición histórica se produjo durante las primeras dos décadas del siglo XX y supuso el paso desde el contexto imperial hacia la tradición nacional.

${ }^{15}$ Aunque ello no necesariamente implique un consenso teórico en cuanto a lo que se entiende por transnacionalismo y el modo de abordarlo, sí puede detectarse que se encuentra muy presente como lógica de fondo que desea trascender el marco y el contexto del Estado-nación como contenedor de análisis. 
cercanos a las perspectivas decoloniales. ¿Cómo se puede traspasar más allá del límite euro-americano y del monopolio epistemológico?... Algunas voces en la última década han reivindicado la necesidad de decolonizar las disciplinas ${ }^{16}$, y buscar las formas posibles de incorporar de modo real y efectivo otras epistemologías. Ello se plantea no sólo como formulación teórica y metanarrativas compartidas y difundidas desde un número cada vez mayor de departamentos de universidades tanto en América como en Europa, sino igualmente a partir de la búsqueda de prácticas metodológicas efectivamente dialógicas y reflexivas.

Si aceptamos dichas premisas como adecuadas, parecería necesario preguntarnos si la antropología social puede, quiere y está dispuesta a iniciar dicho viaje, o en qué medida lo ha iniciado ya.

La condición de crisis no es nueva para la antropología social. Por el contrario, sería un ejercicio imposible de descubrir cuándo la antropología social no ha estado en crisis. La condición de crisis permanente remite en gran parte a casi un continuum que habita la disciplina entre la vulnerabilidad y fragilidad, consustancial a la gran mayoría de las ciencias periféricas. Pero tratemos de imaginar más allá de la respuesta inmediata de éxodo frente a la crisis y pensemos en las posibles tentativas de transformación que los escenarios de crisis pueden proponer.

Haciendo un poco más compleja la última transición histórica de transnacionalización, planteada por Gingrich, nos parece sugerente la propuesta formulada por Santos (2006) de la necesidad de producir teorías y prácticas transescalares; donde las escalas locales se articulan con las escalas nacionales y las globales. En este sentido, entendemos que el reto de la antropología social debería estar en articular las diferentes escalas en los análisis producidos. Como subraya Santos (2006), diferentes experiencias sociales apuntan que las trayectorias son distintas y nunca lineales, y algunas experiencias pueden transitar entre lo local, lo global y después lo nacional ${ }^{17}$. El reto radicaría en ser capaces de incorporar en nuestros análisis y explicaciones las relaciones transescalares, que funcionan como claves para comprender, situar y contextualizar en coordenadas espacio-temporales, por ejemplo, el estudio de los procesos migratorios en el contexto de la globalización.

En una dirección parecida apunta el trabajo de Comarroff (2010), que nos propone el necesario ejercicio de, lo que el autor denomina, la nueva indisciplina: redefinir escalas, conceptos fundacionales y técnicas de producir conocimiento desde la antropología social. De un modo más concreto, para Comarroff (2010), resulta sugerente la propuesta de orientar el futuro de la antropología social hacia una condición indisciplinar, entendida como ese lugar crítico y cuestionador, consecuencia de su permanente estado de crisis, como precisamente potencias intrínsecas.

Comarroff, en su artículo "The End of Anthropology, Again: On the Future of an In/Discipline", identifica tres sintomatologías de crisis. La primera es que la disciplina ha perdido su sello de identidad basado en su método central, la etnografía, sus conceptos estrella — especialmente el de cultura-, su terreno definido como

\footnotetext{
${ }^{16}$ Sobre la idea de decolonizar las ciencias sociales, mirar Walsh (2007).

${ }^{17}$ Es el ejemplo que nos pone Santos sobre los zapatistas (Santos, 2006: 58).
} 
sociedades comparadas y en particular las llamadas sociedades no-Occidentales, y, por último, su campo teórico (Comarroff, 2010: 525). Un segundo síntoma de crisis remite a la inexistencia de un verdadero objeto de investigación, propio de la antropología social — entendido en términos de exclusividad-. Y un tercer síntoma de crisis, según el citado autor, remite a la renuncia de su objeto de estudio, entendido como "sociedades locales" o "culturas", diluyéndose éste en gran parte en todo y nada al mismo tiempo (Comarroff, 2010: 526). A partir de estos síntomas, el autor ha identificado tres tendencias de reacción de la antropología social ante estos escenarios de crisis. Una primera tendencia está siendo volver hacia lo local, la segunda reacción - vinculada con la primera - implica refugiarse en el empirismo fractal (Comarroff, 2010: 528), y una tercera tendencia plantea regresar hacia el concepto de cultura.

Sin embargo, Comarroff cuestiona que seguir adscribiendo el quehacer antropológico a la documentación etnográfica a escala pequeña de culturas no-occidentales, continuar reivindicando dichos enfoques, es en gran parte un anacronismo contraproductivo. Más aún, el autor reivindica la necesidad de comprender la disciplina como práctica, pero entendida en estrecha relación y dependencia teórica.

En pocas palabras, la solución para el autor reside en mapear los procesos de "estar siendo y de convertirse" - process of being and-becoming - a partir de escalas espacio-temporales —aquí y allí, y entonces y ahora-, buscando siempre los contrapuntos entre lo empírico y lo teórico. Estos serían los fundamentos para una antropología crítica e indisciplinada, que presenta según Comarroff, enormes potencialidades para el futuro.

Dentro del mismo número de la revista American Anthropologist, Hannerz (2010) sostiene que fuera de la antropología existe una gran ambigüedad sobre ella, sus temas de investigación y, en definitiva, sus principales características. En su artículo "Diversity is our business", reivindica utilizar la diversidad como elemento clave que permita fabricar una marca identitaria, que puede ayudar a la disciplina a construir una mejor representación de sí misma hacia el exterior. Más aún, reivindica la necesidad de elaborar realmente trabajos comparativos, que procedan de distintos autores y distintas experiencias etnográficas y que permitan llevar a cabo un trabajo real de comparación, muy poco desarrollado en términos absolutos en la actualidad.

De lo mencionado, nos surgen algunos retos a los que estamos tratando de dar respuesta desde nuestras prácticas, y ya estamos en condiciones de producir algunas reflexiones. Nos parece ver que una de las paradojas que nos presenta la permanente crisis de la disciplina - resulta probable que sea de todas las disciplinas - es que en el análisis de sus problemas, de sus crisis, las soluciones que se adivinan pasan por abandonar la "disciplina". La reflexión necesita explicación. Se trata de indisciplinar nuestra producción de saberes, no de someterlos o, al menos, no de exclusivizarlos. En este terreno, sería bueno analizar en la antropología las razones y resistencias para la creación de equipos de investigación, no sólo de grupos, en el estudio de un mismo fenómeno, frente a la tradicional estrategia de robinsones estudiando y produciendo con la soledad del trabajo de campo el conocimiento antropológico. 
Desde estas páginas, nos parece totalmente pertinente la necesidad de reivindicar un prisma transnacional, comparativo y transescalar (Santos, 2006). Sin embargo, estos prismas tendrán poca efectividad si continuamos anclados en el andamiaje del conocimiento disciplinado y no focalizamos nuestros esfuerzos hacia apuestas transdisciplinares. Comprendemos la transdisciplinariedad como las estrategias por medio de las cuales tratamos de poner a conversar múltiples saberes. Es un ejercicio en sí mismo de construir puentes y vasos comunicantes entre distintas formas de comprender y construir conocimientos. Entendemos que nuestras futuras indagaciones tienen que ayudarnos a caminar hacia una transdisciplinariedad, que nos permita abandonar la práctica de disciplinar el conocimiento (Castro-Gómez, 2007) y dejar de organizarlo dentro de las rúbricas correspondientes a estructuras departamentales en la mayoría de las universidades (Gordon, 2006: 3). La voluntad de conseguir la transdisciplinariedad sería posiblemente uno de los pasos que nos permitirá cuestionar la epistemología euro-americana; paso imprescindible para buscar los entrecruces necesarios que hagan posible los diálogos entre saberes. Pero estos procesos dialógicos no sólo deben producirse entre disciplinas centrales y periféricas, sino igualmente entre epistemologías no reconocidas como interlocutoras válidas en las lógicas de producción de conocimiento.

Esto supondrá, para aportar un ejemplo, pasar de una etnografía localizada normalmente en un territorio a ser capaces de analizar las dinámicas transescalares. Se trata de seguir interpretando los múltiples significados de las acciones humanas en los contextos de producción, pero observando que ahora muchas de esas acciones no se sitúan en un solo lugar y que ellas mismas se interconectan entre lugares a veces nada cercanos. El estudio de las migraciones nos está aportando este marco de reflexiones en la medida en que nos muestra que los contextos, esos que con razón hemos privilegiado tanto en la antropología social, no se sitúan ya en un único territorio.

Quizá de esta manera podamos terminar de entender que estudiar en un lugar no es estudiar dicho lugar, como muchas tradicionales etnografías hicieron en el pasado y hacen aún hoy con pretensión de producción de conocimiento antropológico. Tenemos que seguir insistiendo, estudiamos a través de contextos, pero no estudiamos los contextos en sí mismos.

Las tareas son múltiples, e igual que apostar por la transdisciplinariedad, nos parece imprescindible seguir buscando las conexiones entre los espacios académicos y no académicos, entrecruzar los marcos de sentido producidos desde múltiples escenarios. En ese camino, es probable que la antropología social pueda compartir sus no pocas experiencias ya acumuladas en la tentativa de caminar hacia un prisma transdisciplinar.

Si pensamos en la comprensión de las razones de las migraciones, encontramos un buen ejemplo para entender esta multiposicionalidad del trabajo de campo y con ello una nueva manera de concebir el contexto de estudio. Cómo explicar las migraciones ha supuesto muy habitualmente la interrogación a los migrantes y, en no pocas ocasiones, una observación de los contextos a los que llegan. Son ya varias las teorías sobre las migraciones, que nos indican la necesidad de estudiar los contextos 
de partida para obtener respuestas a los interrogantes citados, y en dichos contextos de partida se convertirán en sujetos clave los "no migrantes". De esta manera, comprender que las personas que no migran forman parte del contexto de estudio de las migraciones nos ofrece un ejemplo magnífico para cuestionarnos los tradicionales conceptos de contextos localizados y situados territorialmente. Veremos en algunos de nuestros registros etnográficos una valoración de este argumento.

\section{Algunas traducciones desde diferentes experiencias investigadoras. Pri- meras tentativas hacia el prisma transescalar}

La primera investigación de referencia remite al proyecto "Diffusion and Contexts of Transnational Migrant Organisations in Europe" (TRAMO) ${ }^{18}$. El reto principal del proyecto ha sido compaginar las teorías de los estudios migratorios y las de la sociología de las organizaciones, incorporando un análisis del contexto multinivel en que se ubican — global, nacional, binacional, local— para analizar las variables que influyen en la creación, difusión y características de las asociaciones transfronterizas — cross-border- ubicadas en Alemania, Reino Unido, Polonia y el Estado Español ${ }^{19}$. En estas páginas nos remitiremos al estudio de caso del equipo de la Universidad de Granada, que ha consistido en la investigación en profundidad de dos asociaciones marroquíes y dos ecuatorianas.

Durante el trabajo de campo con las organizaciones seleccionadas, se realizaron entrevistas a expertos internos - miembros de las organizaciones- y externos y observaciones en actividades de las organizaciones, tanto en el país de destino -Estado Español - como en el de origen - Marruecos y Ecuador-. Para identificar dentro de las categorías propuestas a las organizaciones transfronterizas de migrantes seleccionadas, fue necesario atender a un nivel de factores estructurales/ institucionales y a otro nivel de factores situacionales/variables. Estos dos niveles analíticos influyen en la estructura interna, en las estrategias de las organizaciones de migrantes —objetivos/metas, líneas de trabajo, estrategia general, distribución y movilización de recursos- $\mathrm{y}$ en sus actividades internas y externas.

El estudio tenía por uno de sus objetivos estimar la prevalencia de las organizaciones de migrantes transnacionales dentro del conjunto de las organizaciones de migrantes y detectar los factores de contexto, que animan o dificultan su emergencia, difusión y participación efectiva en los contextos espaciales - entendemos que transescalares - en que se ubican. Para evitar un uso demasiado ambiguo del término transnacionalismo y de las organizaciones de migrantes transnacionales, se ha manejado una definición más específica de organizaciones de migrantes

${ }^{18}$ Este proyecto europeo ha estado dirigido por Ludger Pries de la Universidad de Bochum. En el proyecto han participado el Instituto de Migraciones Internacionales de la Universidad de Oxford, el Centro de investigación sobre Migraciones de la Universidad de Varsovia y el Laboratorio de Estudios Interculturales/Instituto de Migraciones de la Universidad de Granada. El proyecto ha sido financiado por la fundación Volskwagen (2007-2010). En la actualidad se está trabajando en la publicación de un libro que presente el conjunto de la investigación TRAMO.

${ }^{19}$ Es importante subrayar que el equipo de investigación era multidisciplinar, y que sólo el grupo de la Universidad de Granada estaba formado por antropólogos/as sociales. 
transnacionales: aquellas organizaciones con distribución de recursos descentralizada y coordinación fuerte entre ambos contextos de origen y destino.

Desde el estudio de caso realizado por el equipo de la Universidad de Granada para entender cómo las asociaciones, su configuración y labor transfronteriza afectan y son afectados por un contexto económico y socio-político que entendemos como transescalar, situamos a los países donde se insertan dentro de los procesos geopolíticos, que relacionan a cada uno de los dos países de origen con el Estado Español, y a su vez sitúan a los tres países dentro del sistema-mundo capitalista global. Además de la posición crítica o no con los diseños geoestratégicos globales o de la respuesta de las asociaciones ante las consecuencias de los mismos, los ciclos de expansión y contracción de la economía mundial, y sus repercusiones específicas en las economías y sistemas socio-políticos nacionales - por consiguiente, en las oportunidades y los límites institucionales para la participación-, así como en las relaciones económicas binacionales, son fundamentales para entender la configuración de las organizaciones de migrantes transfronterizas y sus actividades y demandas principales.

En el caso concreto de esta investigación, por ejemplo, reivindicar la dimensión transescalar ${ }^{20}$, realizando trabajo de campo en diversos escenarios locales - Quito, Madrid, Tetuán, Tánger, Rabat y Barcelona-, observar y hablar con los actores en múltiples escenarios, permite en gran medida no sólo comparar contextos y escenarios diversos, sino también detectar la articulación entre las lógicas geoestratégicas a nivel global sustentadas en la historia colonial, y de qué modo se vertebran con los factores de contexto - estructurales y situacionales-, condicionadas por las propias trayectorias personales y biográficas de los diferentes actores - a partir de sus capacidades de liderazgo, redes existentes, vínculos personales, etc.- . Tan sólo a partir de estas articulaciones multinivel, podemos aproximarnos a comprender las estrategias y respuestas que las organizaciones de migrantes van activando ante circunstancias concretas.

Otros ejemplos etnográficos serían los estudios iniciados hace ya más de una década sobre la presencia de los hijos e hijas de población extranjera en los sistemas educativos de los llamados "países de acogida". En el contexto andaluz venimos trabajando sobre estas cuestiones en clave de construcción de la diferencia en los últimos años $\mathrm{y}$, con la incorporación de una cierta perspectiva transnacional, observamos que se están alterando de manera importante no sólo nuestras formas de proceder, sino, sobre todo, nuestros modos de pensar el fenómeno.

Podríamos empezar por la propia manera de referirnos al fenómeno y con ello de pensarlo. Hablar de inmigrantes en la escuela parece una solución fácil, pero plantea no pocos problemas, desde los estadísticos - lo más habitual en las fuentes oficiales es ofrecer datos de nacionalidad, no la necesaria información demográfica de nacimiento - a los ideológicos — ies correcto llamar a alguien inmigrante por serlo su

${ }^{20}$ Subrayamos que la dimensión transescalar no fue formulada en estos términos en el diseño del proyecto TRAMO y que los otros estudios de caso no han prestado la misma atención a dicha dimensión. El uso del término transescalar (Santos, 2006) ha surgido especialmente en el análisis del estudio de caso del equipo de la Universidad de Granada. 
progenitor? - Pero preferimos tomar un ejemplo de los que representan las "grandes cruzadas", iniciadas en los sistemas educativos de acogida con estos escolares. Nos referimos a las acciones para enseñar la lengua a esos que llaman inmigrantes y que llegan a la escuela sin conocer o dominar la lengua vehicular de la escuela. Sin ningún tipo de pudor pensamos desde las administraciones educativas que lo más conveniente es "compensar ese déficit" y participamos abiertamente del paradigma de la deprivación cultural. Pero el problema es mucho más complejo. Allí donde observamos una acción para mejorar la escolarización del recién llegado, también podemos hallar un sistema para mantener un status quo de la propia escuela. La diversidad dificulta la normal marcha del aula y lo mejor es enseñar la lengua escolar fuera de aquélla al recién llegado, incorporándole al llegar a ser un "alumno normal". Tal explicación parece racional y lógica, pero una observación del valor de la lengua en una escuela y otra, en la de acogida y en la del origen, muestra que sobre este asunto sobrevuelan otras cuestiones relativas a la construcción nacional. La escuela, encargada de presentar y representar las pertenencias nacionales, utiliza la lengua como uno de sus símbolos "naturales". Allí donde quiere verse sólo un progreso "intelectual" del escolar, se gestiona un complejo mundo de construcción de la ciudadanía en los términos en los que la Ilustración la construyó para el Estado moderno. Pero ello exige estar aqui y mirar allí. Y no se trata sólo de distancia metodológica, sino de comprensión de un contexto de campo, que no se debe ni se puede agotar en la escuela de acogida, sino también en la de origen, alejándonos de la clásica manera de entender el contexto y el trabajo de campo - como es lógico, la escuela se quedará pequeña para entender la escuela y exigirá también salir de ella para interpretar los significados de lo que acontece aqui y allí, en términos escolares-.

Y es que los saberes que atesoramos sobre la escuela desde nuestras tradicionales miradas de campo, localizadas en el caso de los estudios sobre migrantes, nos están mostrando una escena de exclusión, pero no estamos terminando de entender la complejidad de las razones. En el caso de los escolares, llamados latinoamericanos, observamos algunos discursos del profesorado mostrando, a pesar del enorme respeto con que estos escolares dicen tratarles, sus más absolutas frustraciones por pensar que la escuela no es para estos "nuevos alumnos"; quiénes son y de dónde vienen sirven para convertirles en los "malos" de la escuela. Pero, ampliando nuestra mirada hacia saberes construidos más globalmente, desde las escuelas de "origen" de estos "nuevos escolares", encontramos no sólo que las lógicas escolares de "buenos" y "malos" alumnos son las mismas "aqui y alli", sino que esos "escolares malos" en destino podían ser los "escolares buenos" en origen. Una etnografía multilocalizada nos empieza a enseñar una escuela, de aquí y allí, en la que dicha institución vuelve a emerger como lugar de producción nacional. No es una simple exclusión la que se sucede, sino la muestra de que para estar se debe ser y, de no ser así, la etiquetación de malos escolares es lo siguiente que ocurre.

Para ilustrar dichas afirmaciones, tomaremos como referencia una de las experiencias de trabajo de campo, llevadas a cabo en el marco del proyecto de investigación "Multiculturalidad e integración de población inmigrante extranjera en las 
escuelas andaluzas" - ver nota a pie 1-. Uno de los casos investigados, fomentado por el análisis del discurso del profesorado al que aludíamos en líneas anteriores, se centró en los escolares de origen ecuatoriano. De manera general son identificados en la escuela como "buenos alumnos" por su respeto hacia el profesorado, su disposición a asumir el rol de alumno tranquilo o su supuesta cercanía "cultural" y lingüística ${ }^{21}$, pero al mismo tiempo se les cataloga como "malos estudiantes" —al no superar con éxito los objetivos planteados en el currículum-.

Uno de los primeros errores que se cometen al hablar desde aquí de allí es la generalización de situaciones y contextos; ni aquí es homogéneo ni allí tampoco. El poder llevar a cabo trabajo de campo en el "mitificado" allí, nos permitió ver un sistema educativo muy diversificado por zonas territoriales, al igual que ocurre aquí, y a la vez muy similar en cuanto a su estructura y funcionamiento, con clara vocación de producción nacional — se enseña en la lengua reconocida como nacional: el castellano; se canta el himno en todas las escuelas; los niños y niñas se forman en el patio frente a una bandera, etc.--, invisivilización de las minorías nacionales - en el caso de España, dicha actitud se plantea ante el pueblo gitano y, en el caso de Ecuador, son las poblaciones afro-ecuatoriana e indígena las minusvaloradas en el contexto educativo nacional-, valorización del inglés como lengua de prestigio frente a otras lenguas locales o más cercanas —en ambos contextos nacionales el inglés es la lengua extranjera enseñada-, y una preferencia por los centros privados frente a los públicos — aumento de colegios de elites, bilingües, etc.- - Sin embargo, la clave para nuestro trabajo no es comparar las estructuras educativas, si no tratar de entender como éstas son percibidas y vividas por los diferentes actores y cómo los lugares de enunciación, desde los que se emiten dichas valoraciones, ideas y/o percepciones, influyen en el modo de entender, estar y ser en una estructura básica en el seno de los Estados: la escuela.

En un primer momento del trabajo de campo, el acercamiento a las interpretaciones de la realidad escolar que mostraba este alumnado, identificado como inmigrante, su grupo de iguales y sus familias nos planteaba serias contradicciones con el discurso procedente del profesorado de las escuelas de destino.

...me dice que sus sistemas educativos están muy por debajo de los nuestros y eso explicaría el fracaso escolar de esta población que, según él, es general. Al preguntarle si conocía el funcionamiento o la estructura del sistema educativo ecuatoriano, me dice que no lo conoce, pero que no tiene más que ver a los chavales para saber que es peor que el nuestro (Extracto de conversación con el Jefe de Estudios de un centro público. Almería. Diario de Campo 1: 12/11/2007).

Si desde la escuela española recogimos no pocos discursos en la línea de la cita anterior, que argumentaban las "enormes" diferencias entre los sistemas educativos y el bajo nivel del sistema educativo ecuatoriano - argumento no muy bien funda-

${ }^{21}$ Es una supuesta cercanía que tiene mucho que ver con las dinámicas coloniales y las relaciones entre ambas naciones (Ecuador-España). 
mentado-, los chicos y chicas ecuatorianos y sus familias "aquí" nos planteaban otras ideas, como en estas palabras de uno de esos escolares ecuatorianos:

Pues, la educación, la educación es más positiva allá porque hay más respeto en todas las formas, y uno no puede contestarle a un profesor allá, se le habla con respeto y educación, digamos, cuando entras, tienes que pedir permiso de ley, igual que aquí [irónico] hay veces que entran y no dicen nada. Tienes que ser puntual en la hora, y en algunos colegios los lunes se paran todos en el patio y cantan el himno (Extracto de la segunda entrevista con un chico de origen ecuatoriano, 11/05/2009).

Esta idea de respeto, que parece imprimir la escuela "alli", y esa relación con la producción de la nación la veremos recogida en no pocos discursos:

Al preguntarles cómo son las escuelas allí, casi todos coinciden en decir que son mejores, que hay más disciplina, que están más ligadas a "la cultura", ya que cantan el himno y se forman en el patio todas las semanas. Edison, que parece el líder del grupo, dice que la escuela de Ecuador es mucho mejor, hay más respeto y es que la educación para él no es sólo saber matemáticas o química, es mucho más útil para la vida saber comportarse con los demás que saber muchas matemáticas. Plantea una dura crítica hacia la sociedad española, argumentando que nosotros sabremos muchas cosas — se refiere a contenidos académicos - , pero no sabemos respetar a los demás porque él lleva cinco años en España y ha encontrado pocos españoles que muestren respeto hacia los extranjeros (Conversación en el parque con seis alumnos de origen ecuatoriano de varios IES de Almería. Diario de campo 3: 01/06/2009).

Este respeto parece también estar relacionado con la disciplina, como se nos muestra en esta conversación, pero donde aparece esa otra concepción que relaciona la escuela con un lugar de saberes enciclopédicos:

Para el padre de Laura sin duda la educación que recibían sus hijos en Ecuador era mucho mejor, más estricta. La madre dice que es diferente, aunque allí la sentía más cercana y tenía más contacto con los profesores. La tía, que lleva aquí diez años, les discute estas ideas, ella dice que aquí salen mejor preparados (Conversación recogida durante la fiesta de fin de curso de un instituto público de secundaria en Almería; familia de una chica de origen ecuatoriano. Diario de campo 3:05/06/2009).

Un primer análisis de esta información nos evidencia una distancia importante en lo que se considera "educación". Los discursos desde las escuelas de aquí nos aluden rápidamente al aprendizaje de unos contenidos y a la superación de una serie de pruebas para demostrar que se han adquirido tales conocimientos, en las cuales el alumnado de origen ecuatoriano no se presenta como exitoso. Desde el otro lado la educación se vincula más con la disciplina, el respeto hacia el profesorado y con potenciar valores y actitudes que pueden llegar a considerarse más importantes que los contenidos académicos. Pero, para entender mejor estos dilemas, el procedimiento 
sería ver el conjunto de los discursos como producciones a diferentes escalas de un mismo contexto global, que debemos tratar de entender cómo se vertebran.

Pero no es nuestro objetivo, en este artículo, profundizar en estas cuestiones, más bien queremos mostrar que, para estudiar fenómenos concretos relacionados con las migraciones, nos interesa ampliar nuestras visiones más allá del "aqui-alli", como si entendiéramos que en los procesos migratorios hay dos lugares de referencia.

Muchas de las personas entrevistadas nos planteaban múltiples versiones de lo que se entiende como "aqui" —Estado español como país receptor; Almería como lugar de residencia; otras ciudades del Estado español en las que han vivido, los barrios, los vecindarios, las escuelas...; al fin y al cabo, las personas con las que se establecen relaciones, las situaciones por las que se atraviesa y las estructuras sociales son las que van a determinar ese sentir de lo que es "aqui" - y "alli" -Ecuador como país; la costa, la sierra o el oriente como zonas geográficas de procedencia que construyen identidades diferenciadas; Latinoamérica entendida como una gran nación frente a los colonizadores; Quito, Esmeraldas, los barrios, los vecindarios, las escuelas...; al fin y al cabo, es más de lo mismo- . Los lugares en sí como espacios delimitados por fronteras no son más que escenarios cambiantes, en los que la vida va trascurriendo, pero, permitiéndonos la metáfora teatral, la importancia del escenario es compartida con la iluminación, las butacas, los actores, las actrices, el público, la organización, etc. Debemos dejar de "estudiar lugares" para estudiar fenómenos sociales y culturales "en lugares" variados y cambiantes e interrelacionados con las lógicas y dinámicas geopolíticas. Utilicemos este enfoque transescalar en el análisis de algunos detalles más precisos del trabajo de campo.

Así, si para nuestra investigación hemos tomado como lugar de referencia la escuela entendiendo ésta como una estructura básica de producción nacional y en la que se inserta un determinado alumnado denominado extranjero, que es problematizado con respecto al buen funcionamiento de esta institución precisamente por no ser nacional, lo que debemos plantearnos - entre otras cuestiones vinculadas a las lógicas del sistema educativo, a las relaciones que atraviesan dicha estructura, etc.- - es por qué este alumnado está ahí, o mejor dicho, aquí.

Esto nos lleva a ubicarnos en las lógicas de las migraciones internacionales, que a su vez nos obligan a remitirnos a las configuraciones geopolíticas en las que se mueven los lugares de origen y destino. En nuestro caso concreto, las referencias a las relaciones coloniales, postcoloniales e incluo neo-coloniales entre Ecuador y España son ineludibles ${ }^{22}$, e incluir estos aspectos en nuestro análisis nos hace entender que, sin una mirada global de estos asuntos, nuestra investigación quedaría incompleta.

${ }^{22}$ Tras la conquista y dominio ejercido por España durante siglos, los vinculos lingüísticos y religiosos son indudables. Si a ello le sumamos la creencia — por ambas partes_ de la existencia de cierta "cercanía cultural", fundamentada esencialmente en dichos vínculos, u observamos la construcción de políticas migratorias que ofrecen ciertas facilidades a los nacionales de Ecuador para vivir y trabajar en España, nos encontramos ante un escenario con una serie de características muy determinadas, que no debemos ignorar en el análisis, puesto que tienen influencia en las personas y sus decisiones. 
Para el caso concreto que queremos plantear como ejemplo, debemos tener en cuenta que la mayoría de las migraciones internacionales son forzadas para los menores, esto es, la decisión de migrar la toma un familiar adulto y, por ello, reducir nuestra "escala" al discurso del menor, nos dejaría ante una explicación parcial del fenómeno.

Cada vez que me entrevisto con Ricardo, me cuenta una historia diferente sobre su proceso de migración, indudablemente su abuela es una pieza esencial en todo esto - siempre sale a relucir que él está aquí por ella, porque ella lo trajo a él y a su padre, pero él va decorando la narración incluyendo episodios de conflictos familiares graves incluso, a veces, incluyendo detalles que me parecen cercanos a narraciones propias del "realismo mágico"-. En principio, me decía que estaban aquí porque su abuela se vino para librarse de la familia, que la presionaba mucho tras la muerte de su madre - la madre de Ricardo - para que volviera a trabajar en la tienda de su tía y dejara el negocio del campo. En otra ocasión, me comentó que allí la finca ya no daba mucho dinero y que una amiga de su abuela trabajaba en una casa en Almería y cobraba bien, así que ella decidió venir... Otro día, me contó que él y su abuela creían que a su madre la habían envenenado y que alguien cercano quería envenenarlos a ellos también para quedarse con las tierras que la abuela tenía en propiedad, y es por esto que ella decidió marcharse. Definitivamente necesito entrevistarme con la abuela (Extracto de Diario de campo 3: 07/05/2009).

Se hace inevitable, pues, llevar nuestra mirada a los responsables directos de la migración del menor. La lógica del trabajo de campo, impregnada por la legalidad en la que se instrumentalizan las relaciones tutelares hacia los menores, nos llevó a entrevistar al padre de Ricardo, otro actor principal en el proceso migratorio, pero que nosotros percibíamos como "secundario" por entender que había sido otra persona la que inició el viaje y posterior agrupación familiar, aunque éste también tenía su visión sobre las razones que motivaron a la abuela de Ricardo a dejar Ecuador.

Bueno yo creo que la abuelita de Ricardo siempre ha querido marcharse de Ecuador, ya lo hizo cuando era más joven, cuando yo conocí a su hija ella ya estaba en Alemania. Así hay mucha gente en mi país, se van, mandan dinero... pero no están con su familia y eso es lo que yo veo peor, porque la mamá de Ricardo estuvo sola mucho tiempo y me lo decía, que le daba pena tener a su mamá lejos. Pero bueno, las cosas cambian y, cuando la abuela se vino, nos quedamos solos Ricardo y yo se hacía dificil salir adelante... Ella quería llevarse al niño, pero yo no iba a dejar que se lo llevara si no iba yo con él porque un padre debe estar con su hijo... (Extractos de entrevista con el padre de Ricardo, 07/06/2009).

Obsérvese ya como el fenómeno de las migraciones tarde o temprano remite a sistemas de relaciones y alianzas que permiten tejer redes, se establecen de una u otra manera conexiones que debemos ir conociendo, describiendo, entendiendo y, sobre todo, concectando como un todo sistema relacional. Parece claro que el padre de Ricardo ligaba su bienestar a la migración y ésta la obtenia por la migración de su hijo. En este sentido, entendemos que esta historia queda incompleta sin la visión de quienes se quedaron allí y vivieron la partida de un familiar; discurso que 
incluimos para mostrar en otra escala el fenómeno migratorio que estamos tratando de comprender. En esta nueva escala aparecen nuevos actores, pero observaremos como continúan construyéndose conexiones entre todos ellos:

Cuando visité a la familia de la señora Inés en Esmeraldas aún conservaban la tienda de la que ésta nos habló en Almería. Conocí a su tía y a sus primas, su tía es una señora muy mayor que nos atiende amablemente desde el recibidor de una tienda en la que venden de todo y que, como la mayoría de los comercios en Esmeraldas, tiene rejas que separan la calle del local. Nos cuenta que allí ellos no vivían mal, textualmente: "para comer no nos faltó nunca", pero claro, cuando "Inesita" tuvo a su hija, las cosas cambiaron, pues ya tenía que ser responsable y había una boca más que alimentar; así, que ella le buscó varios trabajos, además del de la tienda, pero considera que para Inés no era suficiente, ella quería más y decidió irse a costa de dejar a su hija sola. [Se desprende de su discurso cierto reproche hacia la actitud de su sobrina] (Diario de campo 4: 13/10/2009).

La percepción que de los migrantes se tiene desde quienes no migran o no han tenido intención de hacerlo nos parece de suma importancia en el análisis, pues condiciona las relaciones y, como en este caso, puede hacer cambiar los rumbos vitales de ciertos sujetos. Como mostramos antes, el padre de Ricardo nos comentaba que nunca pensó salir de su país, que si lo hizo fue por las circunstancias en las que se encontraba, cuando se quedó sin el trabajo en la finca de su suegra y además esta se marchó dejándolo sólo con su hijo. Y estas valoraciones. que remiten al abandono y a cierta irresponsabilidad en la actitud de los migrantes, se producen de nuevo desde allí:

Elena, la prima de Inés, tiene un discurso muy duro hacia ésta. Dice que no entiende que se fuera más cuando "hace falta que la gente levante el país, ya que el gobierno no va a hacerlo ni lo ha hecho nunca". Dice que Inés no pensó en ellas, que se marchó y fueron ellas las que tuvieron que hacerse cargo de todo, de la tienda, de una tia que tenían enferma, incluso de su hija, y ellas eran casi niñas [se refiere a ella y a su hermana]. Que mandaba dinero, que llamaba por teléfono una vez a la semana, pero "educar a una niña no se hace con plata" y que "así las cosas pasaron como pasaron" [se refiere al embarazo de la hija de Inés con 16 años]. “QQué ibamos a hacer nosotras si no eramos su madre?" (Diario de campo 4: 13/10/2009).

Aunque en otros discursos, emitidos desde el mismo escenario, encontramos otras visiones:

En el rato del recreo pude hablar con Ana, maestra de una escuela infantil y tia de Ricardo. Hasta ese momento, aunque ella había estado presente en las conversaciones con su madre y su hermana, nunca intervino. Al preguntarle sobre su prima Inés, me comenta que cada uno toma las decisiones que cree mejor en su vida y que ella sabe que no fue fácil para Inés dejar a su hija aquí y a toda su familia. Que en general hay una visión de que lo mejor está fuera de Ecuador, de que se gana más dinero, sobre todo en Europa y Estados Unidos, y puede ser verdad pero no es fácil. Ella sabe que Inés ha tenido que trabajar muy duro para poder llevarse a su nieto y 
al padre de éste a España y que la situación que vive no es cómoda, ella cree que lo hace todo por su nieto (Diario de campo, 14/10/2009).

Todo esto nos lleva a entender la diversidad de posiciones que, ante las migraciones, toman ciertos actores relacionados con cada protagonista y que adoptamos como complementarias, como partes de un mismo prisma de innumerables caras. En este sentido, lo que estaríamos construyendo es un marco explicativo acerca de un fenómeno narrado desde diversos ángulos, pero que podría ser representado de manera más clara, si nos imaginamos las observaciones del escenario de relaciones manejando diferentes escalas. Es, pues, desde estos discursos, desde los que partimos para seguir incorporando al análisis una serie de factores que, implícita o explícitamente, se expresan en ellos y que nos están mostrando una complejidad realmente sugerente para el oficio que desempeñamos. ¿Qué dice, pues, la protagonista de los discursos anteriores?

La señora Inés ${ }^{23}$ nos cuenta que se marchó de su país por primera vez cuando era muy joven, el ser madre soltera en Esmeraldas no era algo muy bien visto y estaba cansada de trabajar en la tienda que regentaba su tía, que para ella era como su madre, y hacerse cargo no sólo de su hija, sino también de sus primas que eran menores. Se armó de valor y decidió marcharse a Alemania, donde sabía por algunas vecinas y amigas que encontraría trabajo sin problemas. Con toda la pena dejó a su niña a cargo de su tía y sus primas y se marchó para hacer dinero. A los ocho años regresó con dinero suficiente para comprar una finca y vivir del campo y del comercio del ganado, de cacao, de café y de maíz... Al morir su hija de un ataque al corazón, con tan sólo veintiún años, la señora Inés decide vender la finca y marcharse a España, donde podría alejarse de los recuerdos y encontrar un trabajo bien pagado para mantener a su nieto y llevárselo con ella (Diario de Campo 4: 15/06/2009).

No nos detendremos en ir profundizando en cada uno de los aspectos que nos resultan imprescindibles para armar esa estructura explicativa transescalar que, a modo de tela de araña, nos teje todo un contexto explicativo, cargado de complejidades $^{24}$, pero sí que apuntaremos algunas claves en este sentido.

Incluir el escenario local del que parte Inés es esencial, y no sólo como ella nos lo narra, sino que tener la posibilidad de conocerlo de forma directa nos permite incluir una visión etic del mismo que, al ponerse en diálogo con lo emic, nos descubre nuevas perspectivas del fenómeno que investigamos:

\footnotetext{
${ }^{23}$ Para asegurar el anonimato de las personas con las que hemos trabajado, hemos cambiado sus nombres.

${ }^{24}$ De las palabras de Inés se desprende una importante presión social a la que estaba sometida en origen - muy vinculada a las expectativas de género- y, si la migración en este sentido suponía una salida a dichas exigencias del entorno, éstas se transformaron en la distancia en otro tipo de requerimientos de carácter más tangible: el envío de remesas. Esto modificó la percepción que, de su familia y de su posición en la misma, tenía Inés. Tambien queremos destacar la importancia que las redes han tenido en su toma de decisiones con respecto a la migración. Si hoy reside en Almería es porque una amiga, que ya estaba aquí, le planteó la idea y le ayudó en su búsqueda de empleo; y, si en un momento dado se marchó a Alemania, lo hizo teniendo en dicho país referentes de su propia ciudad.
} 
Esmeraldas es una ciudad abandona por el gobierno central, casi todas las calles, incluidas las arterias de la ciudad, están sin asfaltar, hay cortes de luz constantes. Viniendo de Quito, este lugar parece otro país o, mejor dicho, otra cara de un mismo país. Ya me lo comentaron tanto Ricardo como su abuela, que tuviera cuidado, que era una ciudad peligrosa y muy diferente a las del resto del país. Reconozco que esperaba encontrarme otra situación en la familia de Inés, resulta que están muy bien situados económicamente y son referentes morales en el seno de su comunidad - casi todas las personas a las que he preguntado por la calle los conocen y me hablan bien de ellos, tienen una tienda en el centro de la ciudad muy frecuentada, realizan reuniones semanales de carácter religioso en el patio de su casa- ¿Pero esto siempre ha sido así?, ¿o la contribución de Inés a la economía familiar ha tenido algo que ver en esta "buena posición”? (Diario de Campo 4, 11/10/2009).

Así, nuestra propia percepción de los escenarios y relaciones que en ellos se establecen nos hace embarcarnos en un estimulante viaje, con constantes idas y venidas, de los significados que se les atribuyen y de las relaciones que se construyen y atraviesan los lugares, entendiéndolos como espacios geográficos concretos.

Entender el escenario local, incluso el familiar dentro de lo local, resulta pues tan necesario como ubicarlos en un espacio nacional, dentro del cual tienen un peso específico - en este caso, Esmeraldas es una de las ciudades más periféricas del Ecuador y no sólo literalmente-. A su vez, dicho escenario nacional debe ser revisado tanto desde sus características socio-históricas, políticas y económicas concretas como desde su posición estratégica con respecto a las dinámicas geopolíticas globales - como ya hemos comentado, teniendo en cuenta dentro de éstas las especificidades que lo vinculan con el país de destino-. Y además hemos de tratar de descifrar las influencias que estos aspectos pueden tener en la trayectoria de Inés, así como en nosotros/as mismos/as.

Sin duda, este caso concreto nos muestra como el aquí y el alli aparecen complementados y dan significado al fenómeno que pretendemos estudiar. No sólo se trata de observar que las causas de las migraciones, desde las perspectivas de los migrantes, son varias, sino de ver cómo el contexto de producción del discurso acomoda y moldea el texto, produciendo varios significados que deben ser interpretados e interconectados sobre la base de los escenarios que hemos descrito anteriormente. No se trata de dilucidar qué quiere decir Ricardo, con sus diversas narraciones acerca de su proceso migratorio, Inés, con su explicación sobre su migración, o su tía, sus primas y su yerno, con sus valoraciones, sino de conjugar todas estas explicaciones y construir sus relaciones. Para ello, se hace relevante construir la relación entre los múltiples escenarios manejándonos en diversas escalas.

\section{Breve coda}

En un reciente texto, publicado en esta misma Revista de Antropología Social, el ya citado George Marcus revisaba, entre otros asuntos, alguna de las ideas relacionadas con la etnografía multisituada que hemos esgrimido aquí para construir la noción de contexto en múltiples lugares. Marcus nos recordaba que: 
... el desafío de la etnografía multi-situada — es decir, que deslocalizarse trastorna las convenciones del "estar ahí" - no conduce a una etnografía meramente móvil, que sigue procesos a través de sitios, sino que evoca a la etnografía en sí misma, compuesta de procesos de conocimiento conectados, rizomáticos y víricos. Consiste en seguir conexiones y relaciones de ideas y mapas o topologías que no están dadas, sino que son encontradas (Marcus, 2008: 33).

Aun a costa de insistir, se trata, repitiendo lo ya dicho, no de visitar múltiples lugares, sino de enlazar e interrelacionar los significados de los contextos que estudiamos y articulan los múltiples lugares interconectados, por medio de la construcción de prismas transescaleres, que nos permiten y facilitan redimensionar y poner en relación a los sujetos y las narraciones producidas. Todo ello rompería con una cierta tradición, aún latente en muchas etnografías que siguen estudiando lugares o personas que se sitúan en espacios concretos y determinados. La intención etnográfica sigue siendo para nosotros la interpretación de las culturas (Wolcott, 1985), pero siempre pendientes de la dimensión relacional. Insistimos, no estudiamos lugares, sino a través de los lugares. Ello obedece a la necesidad de inscribir las prácticas culturales en contextos que permitan producir los significados de éstas, atravesadas por contextos económicos y socio-políticos transescalares, situados e imbricados por procesos geopolíticos y geoestratégicos globales.

\section{Referencias bibliográficas}

AGRELA, Belén

2006 Análisis antropológico de las políticas sociales dirigidas a la población inmigrante. Granada: Departamento de Trabajo Social y Servicios Sociales, Universidad de Granada.

AUTANT-DORIER, Claire

2009 "Saisir les identités en mouvement: parenté et histoires de familles turques en migration”. Revue Européenne des Migrations Internationales, 25, 3, 133-151.

BARTH, Frederich

1969 "Introduction", En F. Barth (ed.), Ethnic Groups and Boundaries. Boston: Little Brown, 9-38.

BECK, Ulrich

2000 "The cosmopolitan perspective: sociology of the second age of modernity". British Journal of Sociology, 51, 79-105.

CASTRO-GÓMEZ, Santiago; GROSFOGUEL, Ramón (Eds.)

2007 El giro decolonial. Reflexiones para una diversidad epistémica más allá del capitalismo global. Bogotá: Iesco-Pensar-Siglo del Hombre Editores.

COHEN, Abner

1969 Custom and Politics in Urban Africa: A Study of Hausa Migrants in Yoruba Towns. London: Allen \& Unwin. 
COMARROFF, John

2010 "The End of Anrhropology, Again: On the Future of an In/Discipline". American Anthropologist, 112, 4: 524:538.

DIÁZ DE RADA, Ángel

2010 Cultura, antropología y otras tonterías. Madrid: Trotta.

GIL, Sandra

2006 Las argucias de la integración. Construcción nacional y gobierno de lo social a través de las políticas de integración de inmigrantes: los casos de Cataluña y Madrid. Tesis Doctoral. Departamento de Cambio Social. Universidad Complutense de Madrid.

GINGRICH, Andre

2010 "Transitions: Notes on Sociocultural Anthropology's Present and Its Transnational Potential". American Anthropologist, 112, 4: 552-562.

GORDON, Lewis Ricardo

2006 Disciplinary Decadence: Living Thought in Trying Times. Boulder: Paradigm Publishers.

GRAVES, Nancy B.; GRAVES, Theodore D.

1974 “Adaptive Strategies in Urban Migration”. Annual Review of Anthropology, 3: 117-151.

GREGORIO, Carmen

2009 "Silvia, ¿quizás tenemos que dejar de hablar de género y migraciones? Transitando por el campo de los estudios migratorios". Gazeta de Antropología, 25/1: 1-20.

HANNERZ, Ulf

2003 "Being there . . . and there ... and there! Reflections on multi-site ethnography". Ethnography, 4, 2: 201-216.

2010 "Diversity Is Our Business". American Anthropologist, 112, 4: 539-551.

MARCUS, George E.

1995 "Ethnography In/Of the Word System: the Emergente of Multi-Sited Ethnography". Annual Review of Anthropology, 24: 95-117.

2008 "El o los fines de la etnografía: del desorden de lo experimental al desorden de lo barroco". Revista de Antropología Social, 17: 27-48.

MORAES MENA, Natalia

2010 Transnacionalismo político y nación: el papel del estado y la sociedad civil migrante en la construcción de la trans-nación uruguaya. Tesis doctoral. Granada: Universidad de Granada.

PERÓ, Davide

2009 "Las movilizaciones políticas de los latinoamericanos en Londres”, en A. Escrivá, A. Bermúdez y N. Moraes (eds.), Migración y Participación Política. Madrid: CSIC, 155-182. 
PETERSON, Jr. John H.

1972 "Assimilation, Separation, and Out-Migration in an American Indian Group". American Anthropologist. New Series, 74, 5: 1286-1295.

SANTAMARÍA, Enrique

2002 La incógnita del Extraño. Una aproximación a la significación sociológica de la “inmigración no comunitaria”. Barcelona: Anthropos.

SANTAMARÍA, Enrique (Ed.)

2008 Retos epistemológicos de las migraciones transnacionales. Barcelona: Anthropos.

SANTOS, Boaventura de Sousa

2006 Renovar la teoría crítica y reinventar la emancipación social (encuentros en Buenos Aires). Red de Bibliotecas Virtuales de Ciencias Sociales de América Latina y el Caribe, de la Red de Centros Miembros de Clacso. http://www.clacso.org.ar/ biblioteca.

SEMIN, Jeanne

2009 L'ethnologue dans les réseaux économiques des femmes migrantes: modes de présence simultanée entre la France et l'Afrique. Revue Européenne des Migrations Internationales, 25, 3: 101-113.

VELASCO MAÍLLO, Honorio

1993 Lecturas de antropología social y cultural. La cultura y las culturas. Madrid: UNED.

VELASCO MAÍLLO, Honorio; DÍAZ DE RADA, Ángel

1999 La lógica de la investigación etnográfica: Un modelo de trabajo para etnógrafos de la escuela. Madrid: Trotta.

VERTOVEC, Steven

2007 "Introduction: New directions in the anthropology of migration and multiculturalism". Ethnic and Racial Studies, 30, 6: 961-978.

WALSH, Catherine

2007 ¿Son posibles unas ciencias sociales/culturales otras? Reflexiones en torno a las espistemologías decoloniales". Nómadas, 26: 102-113.

WIMMER, Andreas; GLICK SCHILLER, Nina

2002 "Methodological nationalism and beyond: nation-state building, migration an the social sciences". Global Networks, 2, 4: 301-334.

2003 "Methodological Nationalism, the Social Sciences, and the Study of Migration: An Essay in Historical Epistemology". International Migration Review, 37, 3: 576-610.

WOLCOTT, Harry F.

1993 "Sobre la intención etnográfica", en H. Velasco Maillo, F. J. García Castaño y A. Díaz de Rada (eds.), Lecturas de antropología para educadores. Madrid: Trotta, 127-144. 